IRA-International Journal of Technology \& Engineering ISSN 2455-4480; Vol.07, Issue 02 (2017)

Pg. no. 46-52

Institute of Research Advances

http://research-advances.org/index.php/IRAJTE

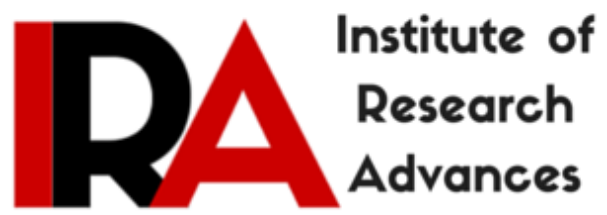

\title{
Swarm Intelligence Based Traffic Control System
}

Bindu Varshini Kosanam

University of Houston, Electrical and Computer Engineering, USA.

AbhinayKukkadapu

Purdue School of Electrical and Computer Engineering, USA.

Type of Reviewed: Peer Reviewed.

DOI: http://dx.doi.org/10.21013/jte.v7.n2.p5

How to cite this paper:

Kosanam, B., \& Kukkadapu, A. (2017). Swarm Intelligence Based Traffic Control System. IRAInternational Journal of Technology \& Engineering (ISSN 2455-4480), 7(2), 46-52. doi:http://dx.doi.org/10.21013/jte.v7.n2.p5

(C) Institute of Research Advances

(oc) EY-NC

This work is licensed under a Creative Commons Attribution-Non Commercial 4.0 International License subject to proper citation to the publication source of the work.

Disclaimer: The scholarly papers as reviewed and published by the Institute of Research Advances (IRA) are the views and opinions of their respective authors and are not the views or opinions of the IRA. The IRA disclaims of any harm or loss caused due to the published content to any party. 


\section{ABSTRACT}

Traffic congestion is one of the major problems in many of metro cities all over the world today. It affects daily life in many aspects such as improper time management, stress, frustration which leads to reckless driving. Average time to reach destination is decided by travel speed which is directly proportional to traffic congestion and also the wait time at each and every traffic signal. In this paper a novel Swarm Intelligence based Traffic Control System (SITS) inspired from nature mimicking (Particle Swarm Optimization) is discussed. This State of the art SITS with Six Degrees of Separation approach to traffic congestion is applying swarm intelligence to traffic lights to avoid waiting of vehicles at red light decreasing wait time and congestion. Here cars will not take random paths or will not take dynamic routes. Six degrees of separation will be employed at each node (traffic signal) to limit computational power and to improve efficiency.
\end{abstract}

Keywords: Swarm Intelligence (SI); Particle Swarm Optimization (PSO); Six degress of separation (SDS); Cloud computing

\title{
INTRODUCTION
}

With the increasing population and with the changing life style the number of vehicles is increasing day to day. To deal with the increasing vehicles on roads effective traffic management systems are very important. One of the main reasons why traffic jams occur is due to improper management of the traffic signals, on many of the roads traffic will be heavy in one specific direction at a given point of time (for example during office hours to reach the office). Even though traffic is heavy in one direction, vehicles in the opposite direction will also have to wait for specific time which is very often more than required at every signal to allow vehicles from other directions, even though there might be few or no vehicles at the perpendicular intersections.

This paper aims to solve this basic and prevailing problem using the data from sensors at traffic signals and connecting them to cloud, which we will discuss in detail in coming sections. This paper introduces a model Particle Swarm optimization to traffic lights (distributed system) using six degrees of separation.

Many papers are published and researches have been done on this topic but the approach is limited to applying swarm intelligence or algorithms on 'vehicles' to find a different route (Ant colony based Swarm intelligence) but Swarm Intelligence based Traffic Control System(SITS) applies same concept on 'Traffic signals'. The information of length of cars that are jammed at a current point (node) at a particular point of time will be dynamically transmitted to cloud. Considering a four-road junction, length of congestion of four directions will be calculated by sensors that are already installed at the roads and transmitted to cloud with a unique id of node.

The main advantage of SITS is leveraging the hardware sensors that are existing at most of the signals in cities. Pooling of this data to traffic signals and transmitting the data from this node to cloud in real-time will help calculate the duration of wait time and making it as null or as minimal as possible is the goal, a detailed scenario will be explained in coming sections.

The term 'Swarm Intelligence' is first coined by Beni and colleagues in their work on cellular robotic systems for the behavior of self-organizing simple agents with the help of interaction with nearest neighbors [1]. Swarm intelligence is an emerging field of scientific research area that involves self-organized, decentralized micro intelligence that leads to global intelligence of entire system. Generally, every system will have a centralized controller which should be sophisticated and this controller will dictate the entire system but on other hand swarm intelligence is unsophisticated smaller systems that cooperate each other to demonstrate desired intelligence to avoid obstacles or to find alternates. This behavior is inspired from nature viz., flock of birds flying, school of fish, colony of ants and in many others [2].

Swarm intelligence is basically cooperating and coordinating with surrounding nodes as well as with the environment. Here each traffic light can be considered as a node and these nodes communicate with each other in a centralized fashion to determine the time for red, green and orange lights. 
Six degrees of separation (SDS) is also an important concept to consider in solving this problem [3]. SDS is an idea that everything of same category (example: humans) are connected by six or less nodes in between. SDS is proposed by Frigyes Karinthy in 1929. In 2001 Duncan Watts from Colombia University, using an email as a package tested this with 48000 senders to reach 19 people which returned an average of intermediaries was around six [11].

For our problem at hand by considering all the nodes in an area will have tremendous number of nodes which will demand very high computational efficiency and processing time which requires a huge software and cloud infrastructure. By using SDS we can now just consider only 6 nodes that are connected and 6 other nodes that are further connected by 6 other nodes and this theory ensures us that all the nodes will be connected.

Considering smaller segments of data would make sense in this case because the traffic at nearby nodes will have huge impact than the nodes that are far away and these smaller systems which decide the timing of the traffic lights will form a huge network of smaller solutions to make an overall solution of a global problem.

Following paper is organized as: II explains the concept of Swarm Intelligence listed as Particle swarm optimization and Six degrees of separation, III explains the concept of Swarm Intelligence based traffic control system (SITS) and its implementation method, IV lists out the differences in approach, advantages and disadvantages. Final section V concludes the observations on SITS

\section{RELATED WORKS}

\section{A. Particle Swarm Optimization}

This algorithm is proposed by Kennedy and Eberhart, it is basically a probabilistic search algorithm. Particles warm optimization, as the name suggests this is an optimization algorithm for combinatorial variable related problems and is more difficult to solve than continuous variable problems as it is time taking.

1) PSO uses velocity vector to update positions of each node in the group or swarm.

2) PSO also acclimatizes to its environment, it is a stochastic process and will gain knowledge 'globally' using the memory of each node and will make the decisions accordingly.

In PSO each node updates its position to the other based on the speed but here we will use the data at each node as a sequence of bits that is to be encoded at a traffic signal before it sends to the cloud. Figure 1 is the flowchart representation of PSO algorithm

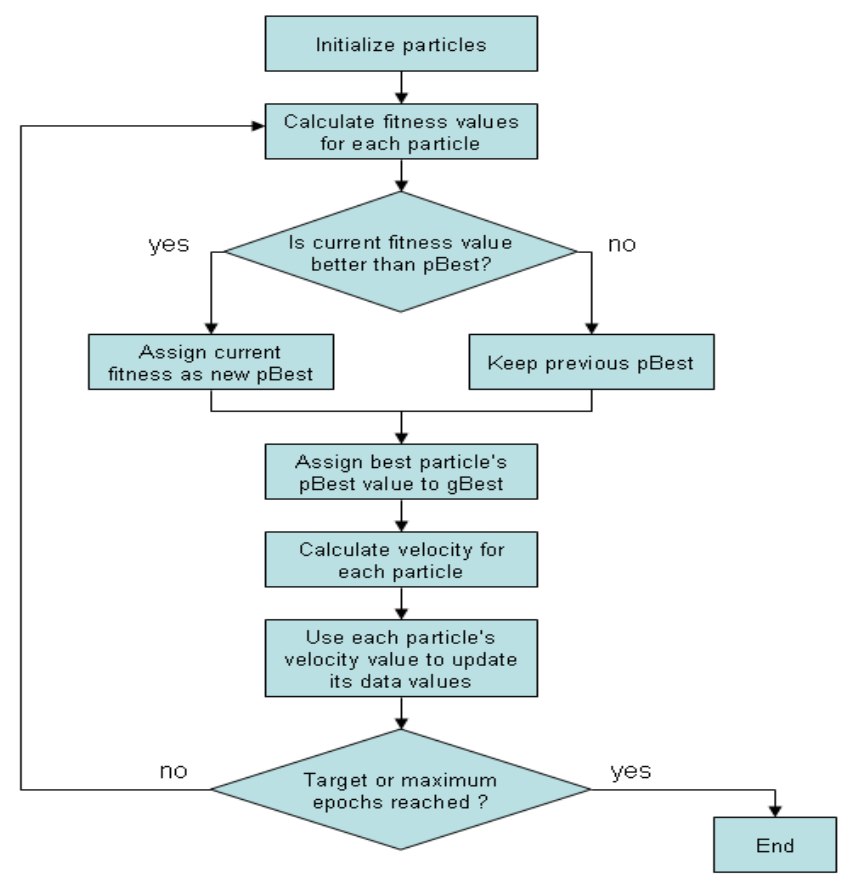

Figure 1. Flow diagram illustrating the particle swarm optimization algorithm[4] 


\section{B. Six degrees of separation}

Six degrees of separation is a theory proposed by Frigyes Karinthy in 1929, that states all living things and everything else in the world is connected by six or less steps in between. This theory is proved by "Small World Project" at Columbia University in 2003.

Bakhshandeh et al conducted an experiment to calculate the average degree of separation between two users on Twitter. Their algorithms turned out to be more efficient and improvement over other greedy algorithms. They found that average degree of separation between two users is 3.43 using their algorithm.

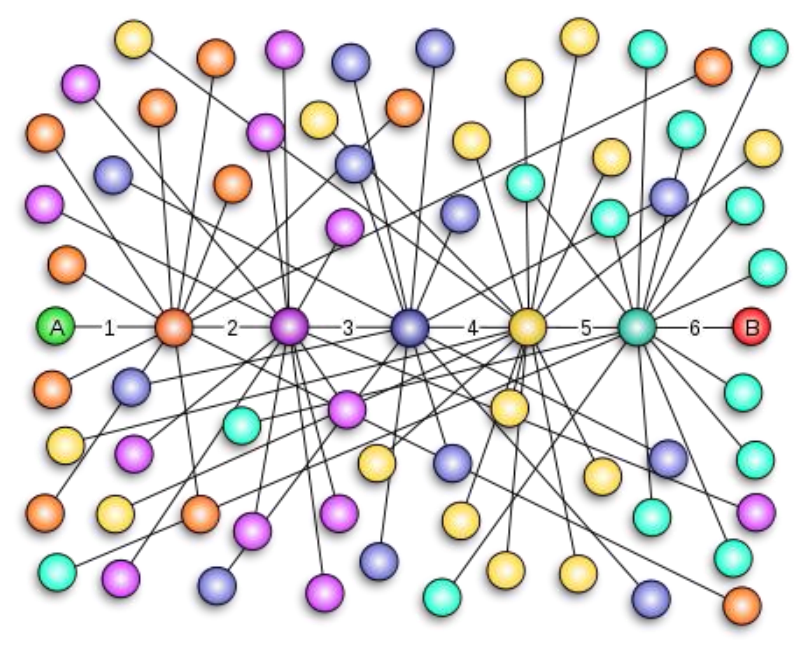

Figure 2. Six Degrees of Separation [5]

From figure 2 each node in this problem space is connected by utmost 6 hops in between. This term is initially coined to signify small world or connected world. SDS is useful to solve our problem of traffic congestion which is explained in detail under SITS model description.

\section{SITS MODEL DESCRIPTION}

There has been a lot of research into the field of Swarm Intelligence and using SI on traffic congestion.

\section{Why Swarm Intelligence?}

Inspired from nature to solve the problem of traffic congestion to reduce wait time at a traffic signal. The core concept of Swarm intelligence is gaining knowledge in their own surroundings (micro level) and assembling all this micro knowledge to solve global problem at hand, i.e., initially the entire system which is distributed all over the problem space is unware of other parts of system [8]. The idea is to connect the individual nodes or collecting knowledge of their surroundings to form a pattern. For example, considering a School of fish or a Flock of birds flying as a group, if the birds that are in the front line see an obstacle, they move away reading the surroundings and all others in the flock will gain that knowledge. Swarm Intelligence works by reading the state of both, fellow nodes and the environment around it.

\section{Why Six Degrees of Separation?}

Considering to solve the problem of traffic congestion, each node is a traffic signal, as there are enormous number of traffic signals in each city processing the data from all these signals is not a plausible solution so as discussed in section II.b SDS assures that all the nodes are theoretically connected and only processing data of six nodes around each node to take a decision is sufficient, saving a lot of computational efficiency. It also makes sense because there is no need to calculate how traffic is at a point that is very far from the current node it may have a less or no impact [5].

\section{E. Concept of SITS using PSO and SDS}

The core idea is to save the time that is spent at the traffic signals, signaled red. Consider a four junction road that has traffic in only in one direction, if the traffic signal has the knowledge of entire 
surroundings around it and the nearby traffic signals and the surrounding of that signal too, then the signal will take its own decision to allow the flow of traffic and the amount of time that it should allow the traffic to flow will also be decided [6].

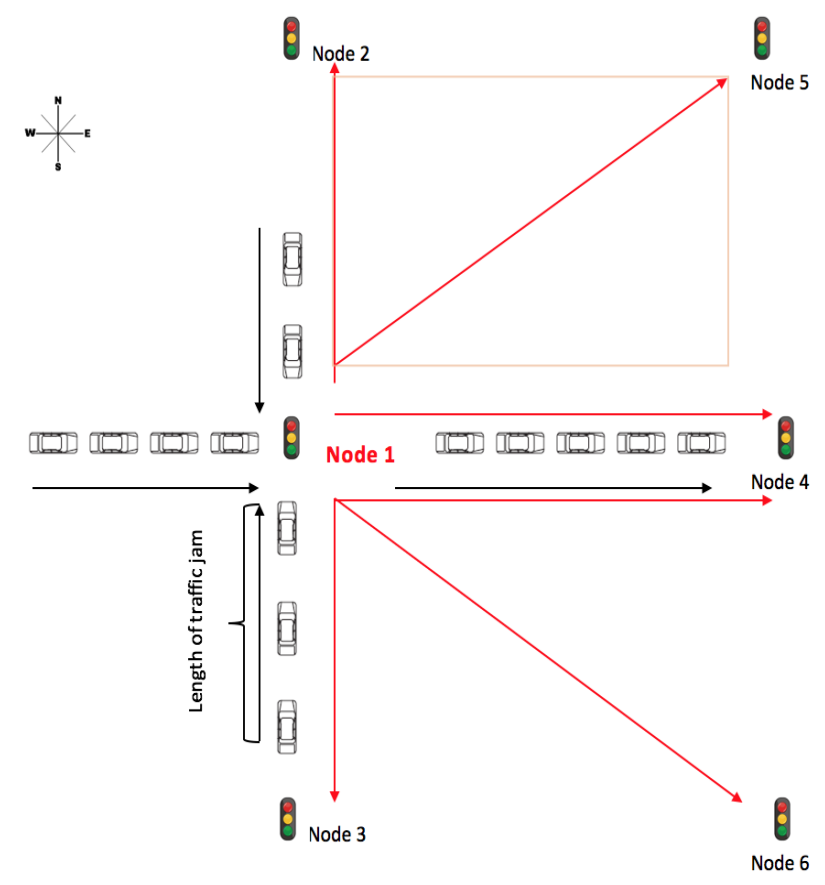

Figure 3. Practical scenario of using SITS concept

Node 1 is where we have to calculate the timing at the signal that it should follow. The sensors that are installed at respective nodes calculate the length of cars that are currently jammed in North, South, East and West directions and includes the information of currently available routes like a routing table at the node, includes information of current estimated time by calculating traffic flow. Each node divides its allocated time to different directions maximizing vehicle flow and minimizing congestion.

Node 1 communicates with surrounding other nodes to calculate the estimated traffic flow in the incoming direction of traffic for node 1 (East). Since each node connects to cloud all the computing takes place in the cloud, just sending the decisions to traffic signals to implement the decision [7].

In figure 3, Node 6'sincoming traffic is effected by Nodes 1,2,3. To effectively distribute the traffic flow of the system, when Node 6 allows the traffic to flow in East Direction Node 1 allows its traffic in North and South (That time will be decided by the calculations in the cloud). When Node 6's outgoing traffic completes in East direction, Node 1 releases its traffic to East. When the traffic from Node 1 reaches Node 6 again they will see immediate green or with minimal wait time, hence minimizing the problem of traffic congestion.

\section{Format OF TrANSMisSion To ClOUd}

A packet of data will be transmitted to cloud that actually calculates the variables that the traffic signal should follow.

\begin{tabular}{|c|c|c|c|c|c|c|c|c|c|}
\hline $\mathrm{L}_{\text {North }}$ & $\mathrm{L}_{\text {South }}$ & $\mathrm{L}_{\text {East }}$ & $\mathrm{L}_{\text {West }}$ & $\begin{array}{l}\text { Ti- } \\
\text { me } \\
\text { tak- } \\
\text { en to } \\
\text { tra- } \\
\text { v-el } \\
\text { las-t } \\
\text { time }\end{array}$ & $\begin{array}{l}\text { Unique } \\
\text { ID } \\
\text { of Node }\end{array}$ & $\begin{array}{l}\text { Are all } \\
\text { roads } \\
\text { active? }\end{array}$ & $\begin{array}{l}\text { Number of } \\
\text { surrounding } \\
\text { nodes }\end{array}$ & $\begin{array}{c}\text { Current } \\
\text { Timestamp }\end{array}$ & $\begin{array}{l}\text { Number } \\
\text { of roads } \\
\text { in the } \\
\text { junction }\end{array}$ \\
\hline
\end{tabular}

Figure 4. Packet Format for transmission 


$$
\begin{aligned}
& \mathrm{L}_{\text {North }} \text { - Length of Traffic in North Direction } \\
& \mathrm{L}_{\text {South }} \text { - Length of Traffic in South Direction } \\
& \mathrm{L}_{\text {East }}-\text { Length of Traffic in East Direction } \\
& \mathrm{L}_{\text {West }} \text { - Length of Traffic in West Direction }
\end{aligned}
$$

\section{ADVANTAGES AND DisdVantages OF SITS}

\section{F. Advantage}

1) Nature mimicking proves that the model is working and can be implemented [9]

2) Very high computational efficiency because of the reuse of knowledge on the nodes

3) Lower number of node processing is adequate between any two nodes because of SDS implementation

4) Effect of any stalled vehicle or any blockage of roads will be propagated down the line to many signals representing the effect and adjusting the timings accordingly and dynamically by which the SITS assures no traffic blockage

5) There are already installed sensors (hardware) that is needed to get the length calculations at the signals which is a huge levarage

6) System is evolvable, adaptation can be shifted from one point to another point and the system dynamically adapts

\section{G. Disadvantages:}

1) There is no central controller for the system due to that there might be some redundancy, cloud is only for processing the data[10]

2) Installing cloud transmission support on traffic signals

3) Managing the cloud for entire city (for traffic lights)

4) Sometimes propagation of effect might take longer due to resolving problems at micro level to get a decision i.e., data getting through different hops.

5) Changes in policies or rules might effect the enire behaviour of system

6) Action of choice is stochastic so behaviour might look noisy

\section{CONCLUSION}

Increasing population and traffic needs demand sophisticated algorithms for traffic control rather than static timings on the signals. In this paper, we outlined a theoretical method that uses Nature mimicking (Swarm Intelligence) technique Particle Swarm Optimization and using Six degree of separation method to increase reliability and improve efficiency. There are complications to adapt to this system but advantages outweigh the complications. Implementing this system assures to solve traffic congestion in heavy traffic areas.

\section{REFERENCES}

[1] Beni, G., Wang, J. Swarm Intelligence in Cellular Robotic Systems, Proceed. NATO Advanced Workshop on Robots and Biological Systems, Tuscany, Italy, June 26-30 (1989)

[2] Reynolds, Craig (1987). "Flocks, herds and schools: A distributed behavioral model.". SIGGRAPH '87: Proceedings of the 14th annual conference on Computer graphics and interactive techniques. Association for Computing Machinery: 2534. doi:10.1145/37401.37406. ISBN 0-89791-227-6.

[3] Newman, Mark, Albert-László Barabási, and Duncan J. Watts. 2006. The Structure and Dynamics of Networks. Princeton, NJ: Princeton University Press.

[4] John McCullock, "Particle Swarm optimization" http://mnemstudio.org/particle-swarmintroduction.htm 
[5] Karinthy, Frigyes. Chain-Links. Translated from Hungarian and annotated by Adam Makkai and Enikö Jankó.

[6] Mu Jun, "Swarm Intelligence Techniques for Route PlanningSystem", Final Year Project, Department of Electrical andComputer Engineering, National University of Singapore, March

[7] Priemer, C., Friedrich, B.: A decentralized adaptive traffic signal control using v2i communication data. In: 12th International IEEE Conference on Intelligent Transportation Systems, ITSC 2009, pp. 1-6. IEEE (2009)

[8] Bonabeau, E., Dorigo, M., Theraulaz, G.: Swarm intelligence: from natural to artificial systems, vol. 1. Oxford University Press (1999)

[9] Sims, A.G.: The sydney coordinated adaptive traffic system. In: Engineering Foundation Conference on Research Directions in Computer Control of Urban TrafficSystems, 1979, Pacific Grove, California, USA (1979)

[10] Mizuno, K., Fukui, Y., Nishihara, S.: Urban traffic signal control based on distributed constraint satisfaction. In: Hawaii International Conference on SystemSciences, Proceedings of the 41st Annual, pp. 65-65. IEEE (2008)

[11] https://en.wikipedia.org/wiki/Six_degrees_of_separation 